\title{
Materialism and the Experience of Flow
}

\author{
Amy Isham ${ }^{1}$ (D) Birgitta Gatersleben ${ }^{2} \cdot$ Tim Jackson $^{1}$
}

Published online: 17 July 2020

(C) The Author(s) 2020

\begin{abstract}
The need to locate ways of living that can be both beneficial to personal well-being and ecologically sustainable is becoming increasingly important. Flow experiences show promise for the achievement of personal and ecological well-being. However, it is not yet understood how the materialistic values promoted by our consumer cultures may impact our ability to experience flow. A cross-sectional survey of 451 people demonstrated that materialistic values and an individual's tendency to experience flow were negatively correlated (Study 1). Next we showed that experimentally priming a materialistic mind-set led to poorer quality flow experiences in a sample of students (Study 2) and British adults (Study 3). Our findings add to current understandings of the detrimental consequences of materialistic values and suggest that it is crucial to challenge the materialistic values present within our consumer societies if we are to provide opportunities for experiencing flow.
\end{abstract}

Keywords Flow $\cdot$ Materialism $\cdot$ Well-being $\cdot$ Sustainability

\section{Introduction}

There is increasing awareness that the world is currently facing a number of environmental issues such as climate change, biodiversity loss, and depletion of natural resources (IPCC 2018; IPBES 2019). Given this, the United Nations' (2015) Sustainable Development Goals highlight the need to create a world in which individuals can thrive whilst limiting environmental damage. Flow experiences have been linked to greater well-being and slightly lower environmental impacts. Testing factors that could influence our likelihood of experiencing flow would therefore be valuable. There is reason to believe that, on top of being linked to poorer well-being and greater ecological footprints, strong materialistic values may undermine the tendency to experience flow. In this research we undertake a direct examination of whether strong materialistic values can undermine our ability to experience flow. This research is important because it suggests, critically, that consumer culture may fundamentally limit our ability to transition towards more rewarding, sustainable lifestyles.

Amy Isham

a.isham@surrey.ac.uk

1 Centre for the Understanding of Sustainable Prosperity, University of Surrey, Guildford GU2 7XH, UK

2 School of Psychology, University of Surrey, Guildford GU2 7XH, UK 


\subsection{Materialism}

Materialism describes a focus on money and material possessions. Most empirical research chooses to consider materialism as a set of values or goals. A value describes "a centrally held, enduring belief which guides actions and judgments" (Rokeach 1973, p. 161). As a value, materialism is proposed to be made up of three sub-components (Richins and Dawson 1992). Firstly, individuals believe that material possessions should be at the centre of their lives, hence granting them the greatest amount of attention, and considering their acquisition to be of the upmost importance (acquisition centrality). Secondly, individuals view the attainment of material items as the key means by which they can improve their own happiness and life satisfaction (acquisitions as the pursuit of happiness). Finally, individuals believe that they should use the ownership of possessions (both number and quality) in order to judge not only their own, but also other people's success (possessions as defining success) (Richins and Dawson 1992). Materialism has sometimes been considered in the context of other life values. For example, Burroughs and Rindfleisch (2002) assessed the relationship of materialistic values as outlined by Richins and Dawson to those life values described within Schwartz's (1992) circumplex value structure. They found that materialism was negatively correlated with collective-oriented values such as benevolence and conformity, and positively related to self-enhancement values such as power and hedonism.

When taking a goal approach, materialism is considered as the greater relative importance placed on extrinsic in comparison to intrinsic goals (Kasser and Ryan 1996). Extrinsic goals are dependent on the reactions of others and usually pursued as a means to end. These include money, fame, and image. Intrinsic goals, on the other hand, are pursued as an end in themselves and often help to satisfy psychological needs as well as aid personal growth. Intrinsic goals include relatedness, helpfulness, and health. When an individual considers extrinsic goals to be more important than intrinsic goals then they may be considered as materialistic.

The strength of materialistic values can be influenced by a number of factors. Greater exposure to consumer advertising (Opree et al. 2014) and growing up with parents who are highly materialistic (Goldberg et al. 2003) have both been linked to stronger materialistic values, for example. Additionally, materialistic values can be manipulated to be more salient in experimental research by exposing participants to materialistic cues such as images of luxury goods (Bauer et al. 2012) or brand logos or advertisements (Whelan and Hingston 2016).

The problematic nature of materialistic values has been documented. Research findings demonstrate that strong materialistic values are associated with poorer well-being, especially for samples of women and those with a higher average age (see Dittmar et al. 2014 for a review). Certain sub-components of materialistic values may be particularly detrimental to well-being. For example, the acquisitions as the pursuit of happiness sub-component has been shown to be the most strongly related to life satisfaction and general well-being (Górnik-Durose 2020; Roberts and Clement 2007; Swinyard et al. 2001). Further, individuals holding stronger materialistic values have been reported to have greater ecological footprints (Brown and Kasser 2005) and to care less about the environment (Hurst et al. 2013). However, how materialistic values may impact upon flow experiences has received relatively little attention in the literature to date. 


\subsection{Flow}

Flow describes a state of optimal experience whereby individuals lose themselves in an attempt to achieve challenging goals. It is typically described as being composed of nine key characteristics, with measures of flow tapping into any number of these. The nine characteristics of flow are as follows. (1) There is a balance between the skills that a challenge requires and those that an individual possesses. The individual is stretched to perform at their highest level but still able to overcome to challenge. (2) The individual is completely concentrated and (3) stops perceiving any barriers between themselves and the activity, giving rise to an experience of effortless movement. (4) Clear goals are present as well as (5) immediate, unambiguous feedback regarding one's progress towards these. The individual (6) feels in control and (7) temporarily loses self-consciousness so can act without fear of judgement or failure. They may experience (8) an altered perception of time, most commonly such that hours seem to go by in minutes. Finally, (9) the experience of flow is intrinsically motivating. This means that people should choose to engage in flow simply because they find it enjoyable, rather than for any external rewards it may bring (Csikszentmihalyi 1975; Jackson and Eklund 2004; Keller and Bless 2008).

Flow can be conceptualised and measured at various levels. Researchers may want to understand the level of flow experienced by someone on a particular occasion or in a particular instance. This is called state flow. Alternatively, they may be interested in which individuals are more prone to experiencing flow (an individual difference-type variable). This is called dispositional flow (or flow proneness) (Jackson and Eklund 2004). Dispositional flow can be assessed for specific activities (Jackson and Eklund 2002) or broader life domains (Ullén et al. 2012).

Flow experiences have been shown to have many benefits for individual well-being. These include higher self-esteem (Hektner and Csikszentmihalyi 1996), greater life-satisfaction (Asakawa 2004) and higher positive affect (Rogatko 2009). Additionally, flow has been suggested to be an important aspect of well-being in itself (Csikszentmihalyi 1990). For example, Seligman's (2012) PERMA theory of well-being outlines five elements that can deliver psychological well-being. These are Positive emotions, Engagement, Relationships, Meaning and Achievement. The engagement element corresponds to the experience of flow. Recent work has also demonstrated that flow experiences may be more likely to occur in activities with lower environmental impacts (Isham et al. 2019). We can therefore see that flow has the potential to promote both individual well-being and environmental sustainability.

\subsection{Materialism and Flow}

It has been suggested that the characteristics associated with the holding of stronger materialistic values may work to hinder an individual's ability to experience flow. Three reasons in particular were highlighted by Kasser (2002). Firstly, materialism is an extrinsic value orientation. That is, individuals' behaviour tends to be focused on external rewards such as money, consumer goods, and fame. This can lead them to be less concerned with an activity itself and hence they fail to fully engage with, or enjoy, it. Their perception is that they are engaging with a task not because they want to, but rather so that they can obtain the reward at the end. Deci, Koestner, and Ryan's (1999) meta-analysis of 128 studies demonstrated that offering rewards for engagement in a behaviour significantly undermined 
intrinsic motivation for that same behaviour. A tendency to view activities purely as a means of obtaining an external reward may therefore undermine the intrinsically motivated nature of flow experiences, via limiting the extent to which people engage with activities for reasons of enjoyment only.

Definitions of materialism emphasize that highly materialistic individuals are concerned with using consumer goods in order to project a certain image (Bauer et al. 2012). Such individuals are therefore likely to be very self-conscious. Schroeder and Dugal (1995) supported this idea by displaying a positive correlation between scores on Ger and Belk's (1996) materialism scale and a measure of public self-consciousness. This extreme focus on how one is perceived by others means that individuals may not grant all of their attention to the activity and hence the experience of flow will be limited.

Finally, materialistic values appear to promote engagement in activities that are unlikely to possess characteristics that are encouraging of the experience of flow. For example television watching has been linked to the possession of stronger materialistic values (Opree et al. 2014), yet this activity has been suggested to be less conducive to flow (Delle Fave and Bassi 2000). Further, Sheldon and Kasser (1995) found that striving for money, fame, and an attractive appearance was associated with greater engagement in behaviours such as watching television as well as smoking and drinking alcohol, which tend to be passive activities requiring little skill. Therefore, people with stronger materialistic values may not choose to engage in activities that have the best potential to facilitate flow, thus limiting their opportunity to have the experience.

Related to the point above, it seems that materialistic values are associated with the choice to engage in somewhat 'easy' behaviours with little challenge. This may be because more materialistic individuals are trying to avoid any emotional discomfort that may come with failing at a challenging task. Numerous studies have demonstrated that materialistic values are associated with the desire to avoid negative emotional states. For example, materialistic values have been positively correlated with experiential avoidance (Kashdan and Breen 2007) and a preference for avoidant coping strategies (e.g. denial, suppression...) after a traumatic event (Somer and Ruvio 2014). Donnelly et al. (2016) even proposed that materialism represents a coping strategy for escaping aversive self-awareness as narrowing attention down onto only consumer goods prevents attention from drifting onto the self. Research has shown that flow experiences are more likely to occur when individuals approach an activity viewing it as an opportunity for personal growth, rather than avoidance of negative thoughts and emotions (Stenseng et al. 2012). Therefore, a further route through which materialistic values may theoretically be able to limit flow experiences is by promoting the desire to avoid negative emotions, which in turns stops people from seeking challenging opportunities for personal growth and flow.

Whilst there have been proposals suggesting a negative effect of materialistic values on flow experiences (Donnelly et al. 2016; Kasser 2002), there have not yet been any quantitative tests of the relationship. The closest study comes from Khanna and Kasser (2001), who demonstrated that students in the US and India who displayed stronger materialistic values reported feeling more alienated during their work, leisure, and personal relationships than their less materialistic counterparts. An alienated person feels separated from their own experience (Dean 1961), estranged from themselves, and isolated from society (Seeman 1959). Alienation appears to be in direct opposition to the experience of flow where individuals report experiencing total control and an absence of any barriers between the self and the activity. Therefore, there is reason to expect that if individuals holding stronger materialistic values are experiencing greater alienation, then they are also likely to be experiencing less flow. 
The present research undertakes the first direct, quantitative exploration of the effect of materialistic values on flow experiences. We start by considering the relationship at the dispositional level, testing whether people with stronger materialistic values are more or less inclined to experience flow in general. We then go on to use experimental methods to determine whether stronger materialistic values can actually cause people to subsequently report poorer quality flow experiences, at the state level.

\section{Study 1}

Study 1 conducted an online survey which measured participants' materialistic values and general tendency to experience the characteristics of flow in their everyday lives. We hypothesized that materialistic values would be negatively related to levels of dispositional flow. Following Górnik-Durose (2020) and other's findings that the acquisitions as the pursuit of happiness sub-component of materialistic values had the strongest relationship with well-being, for exploratory reasons, we also looked for differences in the size of the associations of the different materialistic value sub-components with dispositional flow.

\subsection{Method}

\subsubsection{Participants}

A total of 451 individuals, recruited via social media and the UK-based University's research participation system, took part in the study. Of these, 129 were male, 319 female, and 3 chose not to specify their gender. Participants mean age was $30(S D=13.73)$. The majority were British (55\%), American (15\%) and German (4\%). The median annual personal income bracket was $£ 10-20,000$ p.a. The study passed an ethical assessment in line with the University's ethical guidelines and informed consent was gained for all individuals before they entered the online survey.

\subsubsection{Measures}

2.1.2.1 Materialistic Values The 15-item version of Richins and Dawson's Material Values Scale (MVS; Richins 2004) measured the three proposed components of materialistic values: acquisition centrality $(\alpha=.67)$, acquisition as the pursuit of happiness $(\alpha=.77)$, and possession-defined success $(\alpha=.75)$. Each subscale contains five items to which participants rate their agreement on a scale from 1 (not at all) to 7 (very much). Scores across the three subscales are averaged to give an overall materialistic values score $(\alpha=.86)$. Example items include "I admire people who own expensive homes, cars, and clothes" (success subscale), "I like a lot of luxury in my life" (centrality subscale), and "I'd be happier if I could afford to buy more things" (happiness subscale). The MVS has been shown to have good reliability, with the 15-item version suggested to have a superior dimensional structure than the original 18-item version (Richins 2004; Richins and Dawson 1992). A confirmatory factor analysis demonstrated that the MVS and its three subcomponents had good convergent validity. Convergent validity is considered confirmed when the $\mathrm{CR}$ value for a construct is above .7 and the AVE for a construct is larger than .5 (Hair et al. 2010). In the present study, $\mathrm{CR}=.78$ and $\mathrm{AVE}=.54$. 
Table 1 Descriptive statistics and inter-correlations between materialistic values and dispositional flow

\begin{tabular}{llrrrrrr}
\hline & \multicolumn{1}{c}{$M$} & $S D$ & 1 & 1.1 & 1.2 & 1.3 \\
\hline 1 & Materialistic values & 39.34 & 9.37 & & & & \\
1.1 & Acquisition centrality & 13.96 & 3.41 & $.81^{* * *}$ & & & \\
1.2 & Happiness dimension & 13.12 & 4.09 & $.82^{* * *}$ & $.47 * *$ & & \\
1.3 & Success dimension & 12.26 & 3.82 & $.85^{* * *}$ & $.59^{* * *}$ & $.52^{* * *}$ & $-.14^{* *}$ \\
2 & Dispositional flow & 3.44 & .45 & $-.19^{* * *}$ & $-.11^{*}$ & $-.21^{* * *}$ & - \\
\hline
\end{tabular}

$* * * p<.001 ; * * p<.01 ; * p<.05$

2.1.2.2 Dispositional Flow The English version of Ullén et al's. (2012) Swedish Flow Proneness Questionnaire (SFPQ) tested how often individuals experienced seven characteristics of flow during their work $(\alpha=.73)$, household chores $(\alpha=.71)$, and leisure time $(\alpha=.75)$. Individuals rate how often it happens that they experience each of seven characteristics of flow (e.g. "you feel completely concentrated?" and "you have a sense of complete control?") on a scale from 1 (never) to 5 (every day, or almost every day). Each of the seven characteristics are assessed using one item; the whole scale therefore includes 21 items. When scores across the three subscales are averaged, this gives a measure of how often individuals tend to experience the characteristics of flow in their everyday lives. The overall scale demonstrated good reliability, $\alpha=.81$. Whilst the original scale asks participants to skip the work section if they are unemployed, we chose to allow students to complete this section, noting instead how often they experienced the seven characteristics during study periods.

\subsubsection{Analysis Plan}

The relationships between variables were first tested using correlations. Multicollinearity tests were used in order to determine whether it was appropriate to conduct a regression analysis to assess the amount of variance in dispositional flow accounted for by each of the three materialistic value sub-components. Multicollinearity was considered to not be a problem if the variance inflation factors (VIF) were less than 4.0, and the tolerance greater than 0.2 (Hair et al. 2010). A step-wise regression was employed in order to determine how much additional variance each of the three sub-components could account for.

\subsection{Results}

Means, standard deviations, and correlations between all constructs are given in Table 1. There was a negative relationship between materialistic values and dispositional flow, $r(451)=-.19(95 \% \mathrm{CI}=[-.28,-.10])$. Tests for equality of correlation coefficients (Lee and Preacher 2013) highlighted that the correlation coefficient between the happiness dimension of materialistic values and dispositional flow was significantly more negative than that of the correlation coefficient between the acquisition centrality component of materialistic values and dispositional flow, $\mathrm{z}=-2.38, p<.01$. None of the other MVS 
Table 2 Hierarchical linear regression analysis examining the relationship between the materialistic value sub-components and dispositional flow in Study 1

\begin{tabular}{lllllllll}
\hline & $t$ & $p$ & $\beta$ & $95 \% \mathrm{CI}$ & $F$ & $d f$ & $p$ & Adj. $\mathrm{R}^{2}$ \\
\hline Step 1 & & & & & 5.94 & 1,449 & .015 & .01 \\
Acquisition centrality & -2.44 & .015 & -.11 & $-.20,-.02$ & & & & \\
Step 2 & & & & & 10.11 & 2,448 & .000 & .04 \\
Acquisition centrality & -0.40 & .692 & -.02 & $-.12, .08$ & & & & \\
Happiness dimension & -3.81 & .000 & -.20 & $-.30,-.10$ & & & & \\
Step 3 & & & & & 7.03 & 3,447 & .000 & .04 \\
Acquisition centrality & -0.04 & .966 & -.00 & $-.12, .12$ & & & & \\
Happiness dimension & -3.34 & .001 & -.19 & $-.30,-.08$ & & & & \\
Success dimension & -0.68 & .498 & -.04 & $-.16, .08$ & & & & \\
\hline
\end{tabular}

$D V$ dispositional flow

dimensions significantly differed from each other in the extent to which they were correlated with dispositional flow.

VIF and tolerance values were all less than 2.0 and greater than .50 , respectively, indicating that we did not have a serious problem with multicollinearity. The results of the step-wise regression (see Table 2) demonstrated that the addition of the happiness dimension sub-component in step 2 created the largest improvement in the $\mathrm{R}^{2}$ value for the model, $\mathrm{R}^{2}$ change $=.03, p<.001$. Indeed, when all three subcomponents were included in step 3 , it was only the happiness dimension sub-component that was significantly related to dispositional flow. This therefore supports the correlational outcomes in showing that the happiness dimension has the strongest negative association with dispositional flow.

\subsection{Discussion}

This first study has offered support for a negative, trait-level relationship between materialistic values and dispositional flow. This relationship appears to be particularly strong for the acquisitions as the pursuit of happiness sub-component of materialistic values. This implies that believing that owning material goods can bring you happiness could make individuals less flow prone in comparison to when they just place a lot of importance on acquiring material objects. However, due to the cross-sectional nature of the study we cannot draw any causal inferences. Does the holding of stronger materialistic values actually lead individuals to be less able to experience flow? This is important to determine because it can tell us whether directly targeting materialistic values can have an effect on people's opportunity to experience flow. Study 2 therefore uses experimental manipulations to determine whether the negative effect of materialistic values on flow experiences can also operate directly, at the state level.

\section{Study 2}

Study 2 aimed to determine whether materialistic values have a causal, negative influence on flow experiences using an experimental design and priming methods. It also included an alternative measure of dispositional flow and an additional measure of materialistic 
values. Here we predicted that increasing the salience of materialistic values would lessen the experience of flow in a subsequent activity period, in comparison to when the salience of materialistic values is reduced.

\subsection{Method}

\subsubsection{Participants}

Forty-four students took part in this experimental study, which was conducted at a university campus based in the south east of England. Forty participants were female and four were male. All participants were undergraduate students at the University, ranging from their first to final year. The majority (53\%) identified as British. The mean age of the sample was $19.86(S D=1.11)$. Psychology undergraduate students $(N=33)$ could receive 2 lab tokens for their participation, students from other disciplines received $£ 5$ in return for their participation. The study passed an ethical assessment in line with the University's ethical guidelines.

\subsubsection{Design}

A between-participants design was utilised whereby the main independent variable was whether participants were in the high or low materialism prime group. The salience of materialistic values was manipulated using an 'Imagination Task' ${ }^{1}$ whereby participants had to imagine what they would do in a specified scenario. These scenarios were adapted from those successfully used by Li, Lim, Tsai, and Jiaqing (2015) to prime materialism in Singaporean students. Some changes were made in order to make the scenarios more appropriate for a UK-based audience. For the high materialism group the scenario involved visiting a new luxury shopping centre and winning a $£ 20,000$ voucher to spend within the centre that day. Participants had to list the items they imagined purchasing with their voucher. For the low materialism comparison group, the scenario involved a visit to a countryside village. Participants had to list the things that they imagined themselves seeing or doing were they to take this trip. We expected that this scenario would be able to lessen the salience of materialistic values following findings that immersion in natural environments can orient people away from extrinsic aspirations (Weinstein et al. 2009). Our own pilot test of 18 individuals (all UK-based students under the age of 25) revealed that those individuals exposed to the shopping centre scenario subsequently displayed stronger extrinsic values such as money, fame and image $(M=24.89)$ than those exposed to the countryside scenario $(M=22.44)$. The effect size revealed a small effect $(d=.33)$, as we only had $17 \%$ power to detect effects, this was not statistically significant. Participants were randomly assigned to either the high or low materialism prime group.

\subsubsection{Measures}

3.1.3.1 Materialistic Values Materialism was measured in two ways. The first is through the 14-item shortened version of the Aspiration Index (AI; Martos et al. 2006). Participants rate how important 14 life goals are to them on a scale of 1 (not at all) to 7 (very). Eight

\footnotetext{
${ }^{1}$ Scenarios used are available from corresponding author upon request.
} 
of these are intrinsic goals (e.g. "To work to make the world a better place" and "To have deep enduring relationships") and six are extrinsic goals (e.g. "To be rich" and "To achieve the "look" I've been after"). Both subscales demonstrated acceptable to good reliability across both time points in this study (extrinsic $\alpha=.81-.89$, intrinsic $\alpha=.75-.84$ ). A relative intrinsic versus extrinsic goal orientation (RIEGO) score was computed by subtracting individuals mean score on the intrinsic items from their mean score on the extrinsic items (Sheldon and Krieger 2014). Higher scores therefore reflected a stronger orientation towards extrinsic, relative to intrinsic, goals. Secondly, 6 items in total were taken from Richins and Dawson's Material Values Scale (MVS; Richins 2004), two to represent each of the three proposed sub-components of materialistic values. In the present study, the six items displayed good reliability across both time points, $\alpha=.85-.90$. The REIGO and MVS scores were positively correlated across both time points of the experiment $(r(44)=.50-.67, p<$ $.001)$.

3.1.3.2 Dispositional Flow Dispositional flow was measured using the Short Dispositional Flow Scale 2 (Short DFS2; Jackson et al. 2008). This 9-item scale includes one item to tap into each of the nine characteristics of flow as outlined by Csikszentmihalyi (1992). These are challenge-skill balance, action-awareness merging, clear goals, unambiguous feedback, concentration on the task at hand, sense of control, loss of self-consciousness, time transformation, and intrinsically rewarding. Participants were asked to rate how often they experienced each characteristic, in general, when previously engaging in the activity they had been assigned to take part in in the experiment, on a scale of 1 (never) to 5 (always). This measure is therefore tapping into each participant's general tendency to experience flow in this type of activity. The scale displayed acceptable reliability in the present study, $\alpha=.71$.

3.1.3.3 State Flow State flow (each individual's experience of flow during the lab activity) was assessed using the State Flow Scale 2 (SFS2; Jackson and Eklund 2002). The SFS2 is a 36-item questionnaire. It taps into the same nine flow characteristics as the short DFS-2 but has four items for each characteristic rather than one. Participants were asked to answer concerning the extent to which they experienced each characteristic in the activity period within the experiment, rather than their experiences in the activity in general. The same 1 (never) to 5 (always) response scale was used as in the Short DFS2. The overall scale $(\alpha=$ $.93)$ as well as each of the nine individual characteristics $(\alpha=.74-.94)$ displayed acceptable to excellent reliability in the present study.

\subsubsection{Procedure}

Each participant was run through the experiment individually. After reading the information sheet and providing their informed consent to take part, they first completed the short dispositional flow scale (DFS-2) and the materialism scales. The order of these questionnaires was always randomised. Next came the imagination task. Participants were asked to imagine themselves in either the shopping centre or countryside scenario and answer the question based on the things that they imagined themselves doing in the scenario. Following completion of the imagination task, participants completed the materialism measures again to assess whether the priming had been effective. After this the activity period began. Participants were randomly allocated to either play the computer game Tetris or do some artwork for $15 \mathrm{~min}$. These activities were chosen following previous research showing them to be supportive of flow experiences (Isham et al. 2019; Moller et al. 2010). 
Individuals were instructed to engage with their activity at the level that felt right for them (e.g. altering levels in Tetris, choosing to colour or draw freehand) in order to encourage the balancing of challenge and skill component of flow. The final part of the experiment involved completing the state flow scale (SFS-2). The whole experiment took approximately 40 min to complete.

\subsection{Results}

\subsubsection{Priming Manipulation Checks}

Materialism scores taken pre and post the imagination task were compared for the high and low materialism prime groups using a 2 (Prime Condition: High vs. Low materialism) $\times 2$ (Time: Before vs. After priming activity) mixed factor ANOVA with repeated measures on the second factor. Significant interaction effects were found when examining materialism using the MVS items $\left(F(1,42)=28.04, p<.001, \eta p^{2}=.40\right)$ and Aspiration Index $(F(1,42)$ $\left.=14.65, p<.001, \eta \mathrm{p}^{2}=.26\right)$, indicating that our imagination tasks had differential effects on materialism scores across conditions. Paired samples $t$ tests (see Table 3 ) revealed that for the high materialism prime group, materialistic values as assessed by the MVS questions significantly increased following the priming activity. The relative strength of extrinsic to intrinsic values also increased slightly although this effect was not statistically significant. For the low materialism prime group, materialistic values were significantly lower following the imagination task when considered from both an MVS and Aspiration Index perspective. The prime therefore appears to have been effective in enhancing the salience of materialistic values for those in the high materialism group and reducing the salience of materialistic values for those in the low materialism group.

\subsubsection{The Influence of Materialistic Values on Flow Experiences}

An independent samples $t$ test demonstrated that those individuals in the high materialism prime group $(M=13.64, S D=2.10)$ reported significantly poorer state flow scores than those in the low materialism group $(M=15.01, S D=2.25), t(42)=2.09, p=.032$, Cohen's $d=.63$ (95\% CI[.02, 1.23]). The effect size statistic indicated a medium sized effect. For exploratory reasons, the differences between the two experimental groups' scores on each of the nine components of flow are shown in Fig 1. This was to see if the effect of priming materialistic values on flow was consistent across the characteristics of flow. The pattern was the same across all characteristics such that participants in the materialism prime group reported lower scores. Differences were small to medium sized across all components apart from intrinsic motivation, but these differences were only significant for the challenge/skill balance component $(t(42)=2.14, p<.05)$.

A hierarchical linear regression analysis that entered priming condition as a second step after the age, gender and dispositional flow variables (see Table 4 for outline of results) revealed that being in the high materialism prime group still significantly predicted poorer state flow scores after controlling for the effects of age, gender, and each individuals general tendency to experience flow in the activity they participated in. The size of the effect of experimental condition on state flow scores in this model was small to medium in size, $f^{2}=.13$. The results demonstrated that the addition of the priming condition predictor led to a significant increase in the $\mathrm{R}^{2}$ value for the model, $\mathrm{R}^{2}$ change $=.08, p<.05$. 


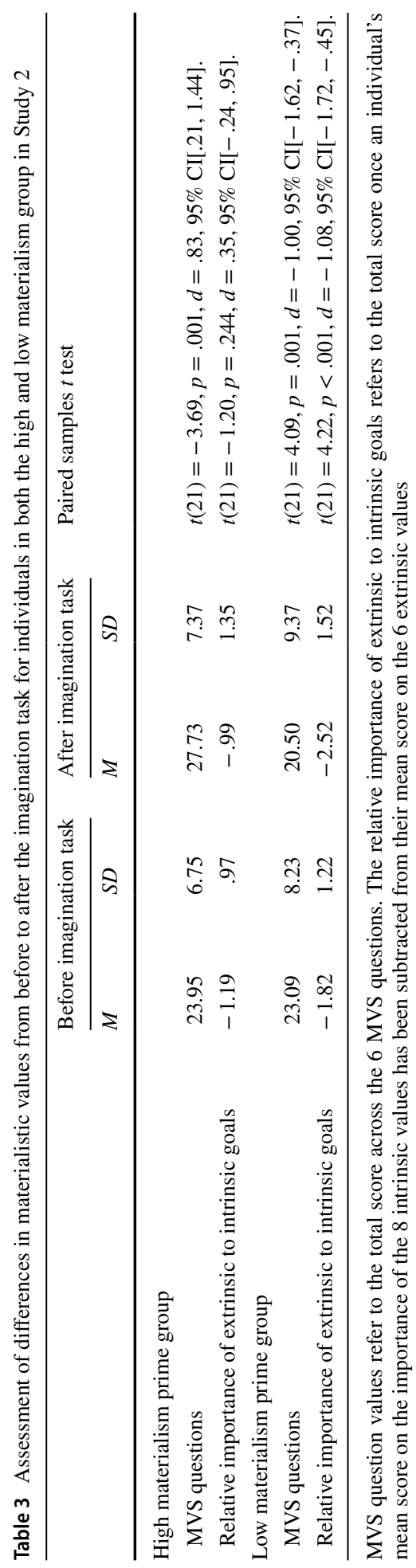




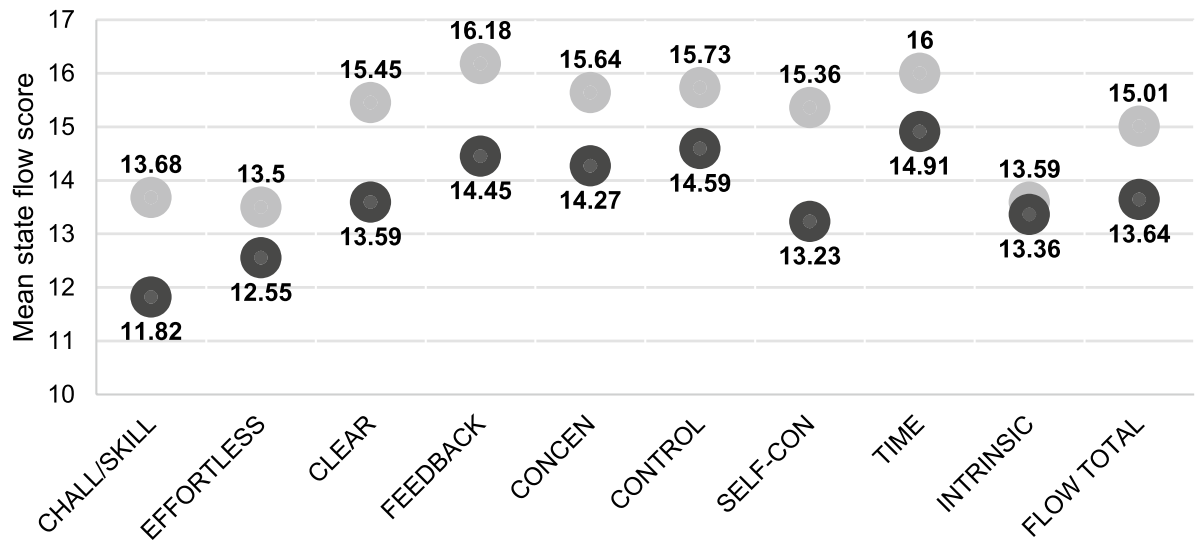

Component of the flow experience

LOW MATERIALISM

HIGH MATERIALISM

Fig. 1 Mean scores for each of the flow sub-components in the high and low materialism prime groups in Study 2. Note that greater scores are indicative of higher flow on all sub-components

Table 4 Hierarchical linear regression analysis examining the influence of the materialism prime in predicting state flow scores in Study 2

\begin{tabular}{lrrrlllll}
\hline & \multicolumn{1}{c}{$t$} & $p$ & $\beta$ & $95 \% \mathrm{CI}$ & \multicolumn{1}{l}{$F$} & $d f$ & $p$ & Adj. $\mathrm{R}^{2}$ \\
\hline Step 1 & & & & & 4.99 & 3,40 & .005 & .22 \\
Age & -1.19 & .239 & -.16 & $-.44, .11$ & & & & \\
Gender $(0=$ male, 1 = female) & .61 & .546 & .08 & $-1.41,2.63$ & & & & \\
Dispositional flow & 3.58 & .001 & .48 & $.21, .76$ & & & & \\
Step 2 & & & & & 5.41 & 4,39 & .001 & .29 \\
Age & -1.42 & .163 & -.18 & $-.45, .08$ & & & & \\
Gender $(0=$ male, 1 = female) & 1.02 & .313 & .14 & $-.13, .40$ & & & & \\
Dispositional flow & 3.52 & .001 & .46 & $.19, .72$ & & & & \\
Prime condition $(0=$ low mate- & -2.26 & .029 & -.30 & $-.56,-.03$ & & & & \\
$\quad$ rialism, 1 = high materialism) & & & & & & & & \\
\hline
\end{tabular}

\subsection{Discussion}

This experimental study has supported our hypothesis that priming a materialistic mindset can act as a causal variable in undermining flow experiences. The negative effect of materialistic values on flow experiences therefore appears to operate at both the trait (Study 1) and state (Study 2) level.

However, as the sample of Study 2 were all university students and mostly female, we decided to run a further experiment in order to test whether the results could be replicated using a more representative adult sample. This was partly to increase the generalisability of our results. It was also partly because a meta-analysis of the relationship between materialism and well-being (Dittmar et al. 2014) documented that age and 
gender exerted moderating effects on the link between materialism and lower personal well-being. This was such that the negative effect of materialism on personal well-being was still negative, but weaker, when the sample was younger and included a larger proportion of males. If materialism has stronger negative consequences for the well-being of different demographic groups, then it could mean that materialism also has greater consequences for the flow experiences of these populations, given that we have outlined flow experiences to be a component of personal well-being. We therefore decided to run a further experiment using an older age sample and an equal proportion of men and women. By comparing the results of Study 2 and Study 3 we could then determine whether the direct, undermining effect of materialism on flow is dependent upon certain participant samples being employed.

In order to gain a better understanding of the size of the negative affect of salient materialistic values on flow quality, we also wanted to employ a neutral control group who did not have the salience of their materialistic values manipulated. In the present study participants either had the salience of their materialistic values strengthened or weakened by the priming activity. Whilst this allowed us to determine whether differing salience of materialistic values can influence flow experiences, it is hard for us to achieve a reliable estimate of the size of these effects unless neutral control groups are present.

Examination of the descriptive statistics presented in Figure 1 revealed that there was not a meaningful difference in intrinsic motivation scores across the two priming groups. However, by instructing participants to engage in a specified activity, this meant that we were potentially undermining the intrinsically motivated aspect of flow for all participants; they were engaging in the activity because they had been instructed to rather than because they wanted to. In Study 3 we therefore wanted to offer participants a choice between three activities to engage in. Self-determination theory states that intrinsic motivation is enhanced when an individual feels in control of his or her actions (Ryan and Deci 2000) and offering choice has been shown to increase feelings of control and intrinsic motivation (Patall et al. 2008). By offering a choice of three activities we hoped to better allow participants to feel free to select an activity that they may enjoy/want to do, hence allowing for intrinsic motivation to potentially occur across all participants.

\section{Study 3}

Study 3 aimed to replicate the findings of Study 2, but using an older sample and a neutral prime that was not intended to influence the salience of materialistic values in the control group. This experiment was conducted online and thus the activities that participants could engage in during the activity period were altered to include tasks that were more easily applied in this context. Participants were also given a choice between three activities to engage in, to facilitate greater intrinsic motivation.

\subsection{Method}

\subsubsection{Participants}

Forty-five individuals took part in this experimental study (23 female, 22 male). Thirtyfour participants were employed, seven were unemployed, and four were retired. All identified as British and were living in the UK. The mean age of the sample was 43.44 ( $S D=$ 
11.99, $\min =26, \max =70$ ). Efforts were made to select equal numbers of individuals across age groups and equal numbers of males and females within each age group. Participants were recruited through the Prolific survey recruitment website and each was reimbursed $£ 1.25$ for their participation in the study.

\subsubsection{Design}

The same between-subjects design was employed as in Study 2. The priming activity for the materialism group was the same shopping centre imagination scenario as used previously. Initially we had wanted to create an imagination scenario for use in a control group, however following difficulties in creating a scenario that did not invoke any particular values, we chose to adopt an image-based control instead. The priming activity for the control group therefore consisted of presenting a number of images that are classified as neutral in valence by the International Affective Picture System (Lang et al. 2008). The same images were used by Bauer et al. (2012, experiment 1) for their control condition when assessing the influence of priming materialism on well-being. ${ }^{2}$ We presented the images as part of a 'recognition memory' task. Participants viewed two sets of 20 images and they had to state whether they had seen each image from the second set in the previous set.

\subsubsection{Measures}

Same as those used in Study 2.

\subsubsection{Procedure}

Each participant completed the experiment online. The order of the experiment was the same as that in Study 2. The only difference was that the activity period lasted for 7 min and participants could choose to engage in either a word search puzzle, guided meditation, or short story writing activity. The word search puzzle was intended to replace the Tetris activity from Study 2 as both activities require visuospatial skills. The short story writing activity was intended to be a creative task, similar to the artwork activity in Study 2. Meditation was selected as the final activity because contemplative activities were highlighted as one of the five groups of 'high flow, low environmental impact' activities outlined by Isham et al. (2019). The other four 'high flow, low environmental impact' activity categories were romantic relationships, creative activities, physical activity, and spending time with others. As flow has been shown to be able to occur across a broad range of activities, we wanted to acknowledge this in the present study. By offering a range of activities that rely on different types of skills we also hoped to increase the likelihood that participants would find an activity that they felt they would enjoy. Research has shown that choice better facilitates intrinsic motivation when respondents have the ability to select an option that is consistent with their own interests (Katz and Assor 2007). A more varied range of activities should

\footnotetext{
2 The reason we did not choose to use the same image-based prime of materialism as Bauer et al. (2012) is because two pilot tests of 20 individuals (one using online opportunity sampling and the other specifically targeting students under the age of 25) that presented the same images of luxury (vs. neutral) goods as part of a 'recognition memory experiment' failed to find any differences in subsequent materialistic values between the materialism and a control prime group.
} 
hopefully mean that a greater number of participants' interests are catered for. Thirtysix participants chose to play the word search, eight to do a guided meditation, and one to write a short story. The whole experiment took approximately 20-25 min to complete.

\subsection{Results}

\subsubsection{Priming Manipulation Checks}

A 2 (Prime Condition: Materialism vs. Control) $\times 2$ (Time: Before vs. After priming activity) mixed factor ANOVA with repeated measures on the second factor compared materialism scores taken pre and post the priming activity for both groups. For the MVS items, the interaction effect was only significant at the $p<.10$ level $\left(F(1,43)=2.78, p<.10, \eta \mathrm{p}^{2}=\right.$ $.06)$, whilst for the Aspiration Index the interaction effect was not significant $(F(1,43)=$ $1.43, n s, \eta \mathrm{p}^{2}=.03$ ). Paired-samples $t$ tests (Table 5) demonstrated that for the materialism prime group, materialistic values as assessed by the MVS questions significantly increased following the priming activity. However, the importance of extrinsic relative to intrinsic goals appeared to be unchanged by the imagination task. For the control group, materialistic values as assessed via the MVS and Aspiration Index were unaffected by the memory task. The primes therefore appear to have been effective in enhancing the salience of materialistic values (as measured by the MVS) for the materialism group only.

\subsubsection{The influence of materialistic values on flow experiences}

Those individuals in the materialism prime group $(M=13.42, S D=1.85)$ reported significantly poorer state flow scores than those in the control group $(M=14.87, S D=2.56)$, $t(43)=2.18, p=.035, d=.65,95 \%$ CI[.05, 1.25]. See Fig. 2 for exploratory differences across the nine flow characteristics. The pattern was the same across all characteristics such that participants in the materialism prime group reported lower scores. Differences were small to large in size across all components apart from an altered sense of time, but were only significant for the challenge/skill balance $(t(43)=2.08, p<.05)$, control $(t(43)$ $=2.14, p<.05)$, and intrinsically rewarding $(t(43)=2.84, p<.01)$ characteristics. Allowing participants to choose the activity they engaged in also appears to have been successful in promoting intrinsic motivation in the control group.

A hierarchical linear regression analysis (Table 6) revealed that there was still a marginally significant effect whereby participants in the materialism prime group reported poorer state flow scores after controlling for age, gender, employment status, and dispositional flow. The size of the effect of experimental condition on state flow scores in this model was small in size, $f^{2}=.09$. The results demonstrated that the addition of the priming condition predictor led to a marginally significant increase in the $\mathrm{R}^{2}$ value for the model, $\mathrm{R}^{2}$ change $=.06, p=.05$.

\subsection{Discussion}

This second experimental study has therefore supported Study 2 in also finding that priming a materialistic mind-set can lead to poorer quality flow experiences when using a sample of British adults. The effect is small to medium-sized, similarly to when a group of 


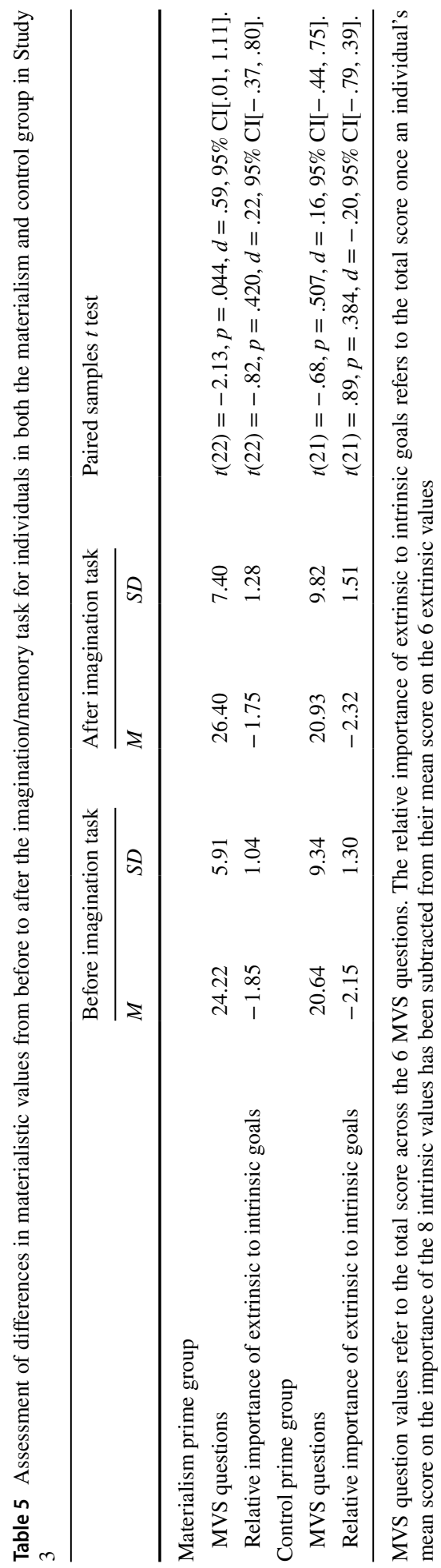



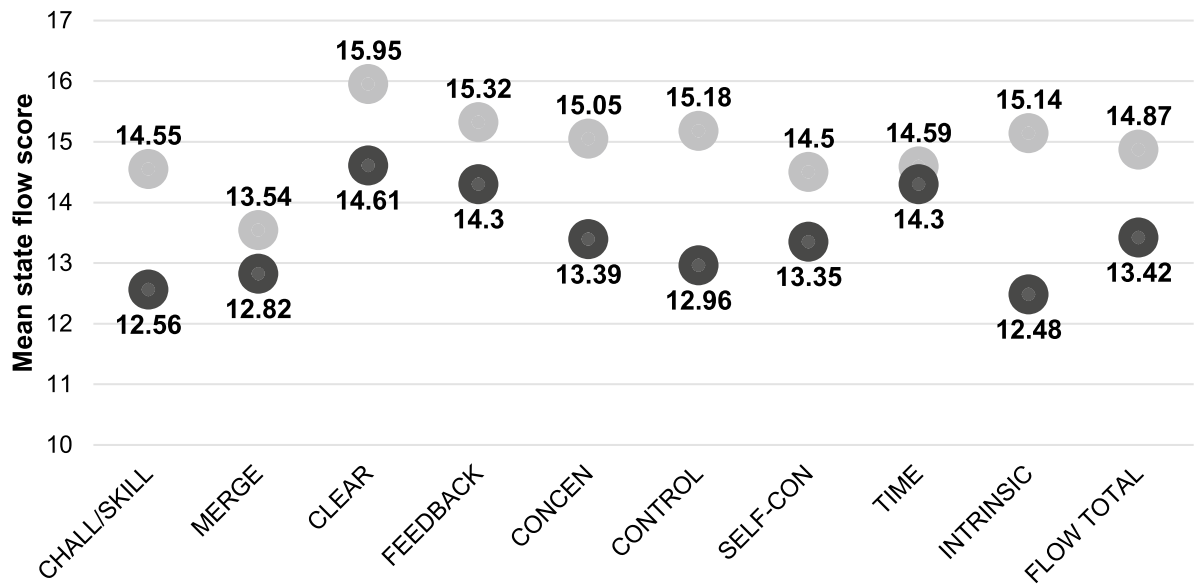

\section{Component of flow experience}

\section{CONTROL OMATERIALISM}

Fig. 2 Mean scores for each of the flow sub-components in the materialism and control prime groups in Study 3. Note that greater scores are indicative of higher flow on all sub-components

Table 6 Hierarchical linear regression analysis examining the influence of the materialism prime on state flow scores in Study 3

\begin{tabular}{|c|c|c|c|c|c|c|c|c|}
\hline & $t$ & $p$ & $\beta$ & $95 \% \mathrm{CI}$ & $F$ & $d f$ & $p$ & Adj. $R^{2}$ \\
\hline Step 1 & & & & & 5.12 & 4,40 & .002 & .27 \\
\hline Age & -.80 & .431 & -.11 & $-.38, .16$ & & & & \\
\hline Gender $(0=$ male, $1=$ female $)$ & -.86 & .397 & -.12 & $-.39, .16$ & & & & \\
\hline $\begin{array}{l}\text { Employment status }(0=\text { unemployed, } 1= \\
\text { employed })\end{array}$ & -.70 & .490 & -.09 & $-.35, .17$ & & & & \\
\hline Dispositional flow & 4.12 & $<.001$ & .57 & $.29, .85$ & & & & \\
\hline Step 2 & & & & & 5.21 & 5,39 & .001 & .32 \\
\hline Age & -.72 & .479 & -.09 & $-.35, .17$ & & & & \\
\hline Gender $(0=$ male, $1=$ female $)$ & -.81 & .426 & -.11 & $-.37, .16$ & & & & \\
\hline $\begin{array}{l}\text { Employment status }(0=\text { unemployed, } 1= \\
\text { employed })\end{array}$ & -.77 & .447 & -.10 & $-.35, .16$ & & & & \\
\hline Dispositional flow & 4.01 & $<.001$ & .54 & $.27, .82$ & & & & \\
\hline $\begin{array}{l}\text { Prime condition }(0=\text { control, } 1=\text { mate- } \\
\text { rialism })\end{array}$ & -2.00 & .052 & -.25 & $-.50, .00$ & & & & \\
\hline
\end{tabular}

predominantly female students were used in Study 2 . The undermining effect of materialistic values on flow experiences therefore also appears to operate within older samples when there is an equal proportion of males and female participants. 


\section{General Discussion}

This research sought to examine whether the materialistic values promoted by our consumer cultures can limit our ability to experience flow, one of the key components of well-being and a potential route towards more sustainable flourishing. Across three studies it has demonstrated that possessing strong materialistic values can limit the extent to which an individual is inclined to experience flow. This is both in terms of the general tendency of individuals with stronger materialistic values to experience the characteristics of flow less often and the direct undermining effect of salient materialistic values on the quality of flow experienced. This research therefore demonstrates that the materialistic values inherent in consumer societies present a significant impediment in the transition to lifestyles that are both rewarding and sustainable.

\subsection{Theoretical Implications}

This research supports previous speculations concerning the negative relationship between materialistic values and the tendency to experience flow (Kasser 2002; Khanna and Kasser 2001). It also allows for certain conclusions concerning Kasser's (2002) three suggestions as to why materialism and flow experiences may be negatively related. The first suggestion was that materialistic individuals are too focused on external rewards (e.g. money and praise) which limits the extent to which they are motivated by enjoyment only (i.e. the intrinsically rewarding aspect of flow). Study 2 failed to document meaningful differences in intrinsic motivation scores across the two experimental groups, but we expected that this may be because instructing participants to partake in a specific activity undermined intrinsic motivation for all participants. When we offered a choice of activities to participants in Study 3, it was found that individuals in the materialism prime group reported finding the activity significantly less intrinsically rewarding than those in the control group. Accordingly, it appears that materialism may partly weaken flow experiences by undermining a focus on intrinsic rewards.

Kasser's (2002) second suggestion was that highly materialistic individuals were too pre-occupied with their public image and that this limited their flow experiences. In both Study 2 and Study 3, there were small differences whereby individuals who had the salience of their materialistic values increased reported poorer scores on the loss of self-consciousness aspect of the flow experience. This therefore supports the idea that thinking about material goods can heighten people's public self-consciousness which in turn hampers their experience of flow.

The last of Kasser's proposals was that individuals possessing strong materialistic values may not choose to engage in activities that are conducive to flow. In Study 2 and 3 participants were offered the chance to participate in an activity that previous studies had shown to be flow-conducive. Those participants with a materialistic mind-set primed still reported poorer state flow scores. Therefore, it appears that it is not just that highly materialistic people may not often take part in flow-supportive activities which prevents them from experiencing flow. Rather, there must also be something more central to the way in which they approach any activity which drives this effect. We are currently conducting further work to determine the specific differences in how more and less materialistic individuals approach activities, but potential factors are that they are more focused on how others are perceiving them, don't stretch themselves to increase 
their skill level, or may lack the ability to regulate attention to maintain focus on the task.

The finding that strong materialistic values are negatively related to the tendency to experience flow may also have implications for the wider literature surrounding the relationship between materialistic values and poor personal well-being. Here we have considered flow to be an important aspect of well-being in itself. However, frequent and/or high quality experiences of flow have also been linked to other aspects of well-being such as self-esteem (Hektner and Csikszentmihalyi 1996), life satisfaction (Asakawa 2004), and eudaimonic well-being (Schwartz and Waterman 2006). A reduced tendency to experience flow may therefore be able to explain partly why we often see individuals holding stronger materialistic values scoring poorly on these various other aspects of well-being (Chang and Arkin 2002; Hudders and Pandelaere 2012; Kasser and Ahuvia 2002).

\subsection{Practical Implications}

Demonstrating that materialistic values can undermine beneficial flow experiences indicates that our consumer capitalist cultures, which present the good life as an affluent one, filled with expensive, consumer goods (Kasser et al. 2007), may work to hamper the very experiences (e.g. flow) that could make life fulfilling and meaningful. Having found that materialistic individuals will report poorer flow experiences even when given the opportunity to engage in a potentially high-flow activity, creating opportunities for flow will not be enough to encourage these experiences in individuals who have already bought into the materialistic ideal. Instead, if we want to provide opportunities for flow, we will need to explore ways of altering the problematic aspects of materialistic values themselves.

Challenging materialistic values will be difficult, especially given that they can be prompted by simply thinking about luxury products or viewing consumer-related images, which are prevalent in our consumer societies. Potential means of reducing the strength of materialistic values include greater exposure to nature (Weinstein et al. 2009) and reduced exposure to advertising (Opree et al. 2014), especially that which depicts images of luxury goods. Indeed, our methods for altering the strength of materialistic values in this research revolved around imagining nature or luxury items. Marketers will be able to have an impact here by considering the content of the advertising-related media we are exposed to. Teachers and parents could also have a role to play as it has been shown that children are less susceptible to advertising and consumer messages if their family regularly discuss advertising and consumption issues (Buijzen and Valkenburg 2003).

In addition to well-being, flow has been shown to benefit many other outcomes including greater academic (Larson 1992) and job performance (Demerouti 2006). Our findings therefore imply that reducing materialistic values could benefit areas such as educational and occupational progress, by allowing for the opportunity for flow experiences in these contexts.

\subsection{Difficulties, Limitations and Future Research}

In this research we have examined the effect of materialistic values on flow. One crucial area for further research is to explore this relationship in the opposite direction. Csikszentmihalyi (2004) proposed that materialistic pursuits represent a coping mechanism used by individuals to fulfil their existential need to keep consciousness ordered and prevent self-rumination. Flow presents a means through which consciousness can 
be focused. When an individual is not able to keep consciousness focused through flow experiences, they may use materialistic pursuits as a substitute, encouraged by the advertising industry in modern consumer cultures. Difficulties experiencing flow may therefore encourage the adoption of stronger materialistic values. If this is found to be the case then providing opportunities for people to create flow across society could be one means of preventing the initial adoption of strong materialistic values.

One of the primary limitations of this research is that the participants were not representative of all socioeconomic backgrounds. It is unlikely that any of our participants were experiencing poverty as our study required that they had access to a computer for use in their own time or that they were undergoing a university education. Research has suggested that individuals from poorer socioeconomic backgrounds tend to score higher on measures of materialism (Chaplin et al. 2014; Sheldon and Kasser 2008) and income has also been positively correlated with dispositional flow (Sahoo and Sahu 2009). Although Dittmar et al.'s (2014) meta-analysis did not find income to moderate the relationship between materialistic values and well-being, if there is a tendency for materialistic values to be higher and opportunities for flow to be limited within very low income groups, then these people may represent a crucial demographic for interventions and therefore should be incorporated into future research.

Ideally, we would have also liked to have had larger samples for the experimental studies. We therefore have to appreciate that our effect sizes may be slightly inflated due to the smaller sample sizes employed here. Nevertheless, as this is the first piece of research to examine the effect of materialistic values on flow experiences, we feel that finding that increasing the salience of materialistic values leads to poorer quality flow experiences across two samples indicates that this is a promising area for further study.

One of the main difficulties when conducting this research was utilising an effective means of priming materialistic values. The imagination task was effective at enhancing the salience of materialistic values. We believe that this was because it required participants to articulate very particular items that they wished to own, in comparison to just viewing images of luxury goods which may have had little personal relevance to them. However, we were unable to devise an imagination scenario that would be valueneutral. Therefore, in order for both the experimental and control condition to have the desired effects on materialistic values, different task types had to be used in Study 3. This may allow for the presence of confounding variables. For example, the memory task may have been more boring than the imagination task. As boredom has been shown to often lead to reductions in effort and attention (Pekrun et al. 2010), this could mean that individuals in the control group went on to be less focused on the flow activity because of the type of priming activity they were previously exposed to. Whilst we demonstrated that those participants in the materialism prime group displayed poorer state flow scores than the control group despite this potential confounding effect, differential effects of task type on the dependent variable are still important to acknowledge when this is not able to be held consistent across the experimental and control group. Relatedly, the materialism prime used in the present study was fairly strong and direct, incorporating a luxury shopping centre and a large amount of money to spend. It may be useful for future research to also incorporate more subtle materialism cues, for example having a smaller amount of money to spend or viewing advertisements for non-luxury products. This would determine the extent to which weaker, but still common, consumer-cues could also influence flow experiences. 


\section{Concluding Remarks}

Existing research suggests that flow experiences offer a promising route for promoting both personal and ecological well-being. However, across three studies we show that individuals holding stronger materialistic values are less likely to report experiencing the characteristics of the flow state. This means that values promoted by consumer cultures may hinder attempts to transition towards more rewarding, sustainable lifestyles, by hampering flow experiences. The identification of a causal, negative link between materialistic values and flow experiences indicates that it is crucial that we directly challenge the materialistic values present within our societies if we are to make progress towards sustainable development targets.

Acknowledgements This research was conducted with support from the Economic and Social Research Council (ESRC) and is part of the interdisciplinary research program of CUSP - the Centre for the Understanding of Sustainable Prosperity. The authors declare that they have no conflict of interest. We are thankful to the three anonymous reviewers for their constructive comments.

Open Access This article is licensed under a Creative Commons Attribution 4.0 International License, which permits use, sharing, adaptation, distribution and reproduction in any medium or format, as long as you give appropriate credit to the original author(s) and the source, provide a link to the Creative Commons licence, and indicate if changes were made. The images or other third party material in this article are included in the article's Creative Commons licence, unless indicated otherwise in a credit line to the material. If material is not included in the article's Creative Commons licence and your intended use is not permitted by statutory regulation or exceeds the permitted use, you will need to obtain permission directly from the copyright holder. To view a copy of this licence, visit http://creativecommons.org/licenses/by/4.0/.

\section{References}

Asakawa, K. (2004). Flow experience and autotelic personality in Japanese college students: How do they experience challenges in daily life? Journal of Happiness Studies, 5(2), 123-154. https://doi. org/10.1023/B:JOHS.0000035915.97836.89.

Bauer, M. A., Wilkie, J. E., Kim, J. K., \& Bodenhausen, G. V. (2012). Cuing consumerism: Situational materialism undermines personal and social well-being. Psychological Science, 23(5), 517-523. https://doi.org/10.1177/0956797611429579.

Brown, K. W., \& Kasser, T. (2005). Are psychological and ecological well-being compatible? The role of values, mindfulness, and lifestyle. Social Indicators Research, 74, 349-368. https://doi.org/10.1007/ s11205-004-8207-8.

Buijzen, M., \& Valkenburg, P. M. (2003). The unintended effects of television advertising: A parentchild survey. Communication Research, 30(5), 483-503. https://doi.org/10.1177/009365020325636 1 .

Burroughs, J. E., \& Rindfleisch, A. (2002). Materialism and well-being: A conflicting values perspective. Journal of Consumer Research, 29(3), 348-370. https://doi.org/10.1086/344429.

Chang, L., \& Arkin, R. M. (2002). Materialism as an attempt to cope with uncertainty. Psychology \& Marketing, 19(5), 389-406. https://doi.org/10.1002/mar.10016.

Chaplin, L. N., Hill, R. P., \& John, D. R. (2014). Poverty and materialism: A look at impoverished versus affluent children. Journal of Public Policy \& Marketing, 33(1), 78-92. https://doi.org/10.1509/ jppm.13.050.

Csikszentmihalyi, M. (1975). Beyond boredom and anxiety. San Francisco, CA: Jossey-Bass.

Csikszentmihalyi, M. (1990). Flow: The psychology of optimal experience. New York: Harper \& Row.

Csikszentmihalyi, M. (1992). Flow: The psychology of happiness. London: Rider.

Csikszentmihalyi, M. (2004). Materialism and the evolution of consciousness. In T. E. Kasser \& A. D. Kanner (Eds.), Psychology and consumer culture: The struggle for a good life in a materialistic world (pp. 91-106). Washington, DC: American Psychological Association. 
Dean, D. G. (1961). Alienation: Its meaning and measurement. American Sociological Review. https:// doi.org/10.2307/2090204.

Deci, E. L., Koestner, R., \& Ryan, R. M. (1999). A meta-analytic review of experiments examining the effects of extrinsic rewards on intrinsic motivation. Psychological Bulletin, 125(6), 627-668. https ://doi.org/10.1037/0033-2909.125.6.627.

Delle Fave, A., \& Bassi, M. (2000). The quality of experience in adolescents' daily lives: Developmental perspectives. Genetic, Social, and General Psychology Monographs, 126(3), 347-367.

Demerouti, E. (2006). Job characteristics, flow, and performance: The moderating role of conscientiousness. Journal of Occupational Health Psychology, 11, 266-280. https://doi. org/10.1037/1076-8998.11.3.266.

Dittmar, H., Bond, R., Hurst, M., \& Kasser, T. (2014). The relationship between materialism and personal well-being: A meta-analysis. Journal of Personality and Social Psychology, 107(5), 879-924. https://doi.org/10.1037/a0037409.

Donnelly, G. E., Ksendzova, M., Howell, R. T., Vohs, K. D., \& Baumeister, R. F. (2016). Buying to blunt negative feelings: Materialistic escape from the self. Review of General Psychology, 20(3), 272-316. https://doi.org/10.1037/gpr0000078.

Ger, G., \& Belk, R. W. (1996). Cross-cultural differences in materialism. Journal of Economic Psychology, 17(1), 55-77. https://doi.org/10.1016/0167-4870(95)00035-6.

Goldberg, M. E., Gorn, G. J., Peracchio, L. A., \& Bamossy, G. (2003). Understanding materialism among youth. Journal of Consumer Psychology, 13(3), 278-288. https://doi.org/10.1207/S1532 7663JCP1303_09.

Górnik-Durose, M. E. (2020). Materialism and well-being revisited: The impact of personality. Journal of Happiness Studies, 21, 305-326. https://doi.org/10.1007/s10902-019-00089-8.

Hair, J., Black, W., Babin, B., \& Anderson, R. (2010). Multivariate data analysis (7th ed.). Upper Saddle River, NJ: Prentice-Hall Inc.

Hektner, J. M., \& Csikszentmihalyi, M. (1996). A longitudinal exploration of flow and intrinsic motivation in adolescents. In: Paper presented at the Annual Meeting of the American Educational Research Association (New York, NY, April 8-12, 1(1996).

Hudders, L., \& Pandelaere, M. (2012). The silver lining of materialism: The impact of luxury consumption on subjective well-being. Journal of Happiness Studies, 13(3), 411-437. https://doi. org/10.1007/s10902-011-9271-9.

Hurst, M., Dittmar, H., Bond, R., \& Kasser, T. (2013). The relationship between materialistic values and environmental attitudes and behaviors: A meta-analysis. Journal of Environmental Psychology, 36, 257-269. https://doi.org/10.1016/j.jenvp.2013.09.003.

IPBES. (2019). Global assessment report on biodiversity and ecosystem services. Bonn, Germany: The Intergovernmental Science-Policy Platform on Biodiversity and Ecosystem Services. https://ipbes .net/global-assessment-report-biodiversity-ecosystem-services.

IPCC. (2018). Global warming of $1.5^{\circ} \mathrm{C}$ (IPCC Special Report). Geneva, Switzerland: Intergovernmental Panel on Climate Change. https://www.ipcc.ch/sr15/.

Isham, A., Gatersleben, B., \& Jackson, T. (2019). Flow activities as a route to living well with less. Environment and Behavior, 51(4), 431-461. https://doi.org/10.1177/0013916518799826.

Jackson, S. A., \& Eklund, R. C. (2002). Assessing flow in physical activity: the flow state scale-2 and dispositional flow scale-2. Journal of Sport \& Exercise Psychology, 24(2), 133-150. https://doi. org/10.1123/jsep.24.2.133.

Jackson, S. A., \& Eklund, R. C. (2004). The flow scales manual. Morgantown, WV: Fitness Information Technology.

Jackson, S. A., Martin, A. J., \& Eklund, R. C. (2008). Long and short measures of flow: The construct validity of the FSS-2, DFS-2, and new brief counterparts. Journal of Sport and Exercise Psychology, 30(5), 561-587. https://doi.org/10.1123/jsep.30.5.561.

Kashdan, T. B., \& Breen, W. E. (2007). Materialism and diminished well-being: Experiential avoidance as a mediating mechanism. Journal of Social and Clinical Psychology, 26(5), 521-539. https://doi. org/10.1521/jscp.2007.26.5.521.

Kasser, T. (2002). The high price of materialism. Cambridge, MA: MIT Press.

Kasser, T., \& Ahuvia, A. (2002). Materialistic values and well-being in business students. European Journal of Social Psychology, 32(1), 137-146. https://doi.org/10.1002/ejsp.85.

Kasser, T., Cohn, S., Kanner, A. D., \& Ryan, R. M. (2007). Some costs of American corporate capitalism: A psychological exploration of value and goal conflicts. Psychological Inquiry, 18(1), 1-22. https://doi.org/10.1080/10478400701386579. 
Kasser, T., \& Ryan, R. M. (1996). Further examining the American dream: Differential correlates of intrinsic and extrinsic goals. Personality and Social Psychology Bulletin, 22(3), 280-287. https:// doi.org/10.1177/0146167296223006.

Katz, I., \& Assor, A. (2007). When choice motivates and when it does not. Educational Psychology Review, 19(4), 429. https://doi.org/10.1007/s10648-006-9027-y.

Keller, J., \& Bless, H. (2008). Flow and regulatory compatibility: An experimental approach to the flow model of intrinsic motivation. Personality and Social Psychology Bulletin, 34(2), 196-209. https:// doi.org/10.1177/0146167207310026.

Khanna, S., \& Kasser, T. (2001). Materialism, objectification, and alienation from a cross-cultural perspective. Unpublished manuscript.

Lang, P. J., Bradley, M. M., \& Cuthbert, B. N. (2008). International Affective Picture System (IAPS): Affective ratings of pictures and instruction manual (Technical Report No. A-8). Gainesville, FL: University of Florida.

Larson, R. (1992). Flow and writing. In M. Csikszentmihalyi \& I. S. Csikszentmihalyi (Eds.), Optimal experience: Psychological studies of flow in consciousness (pp. 150-171). New York: Cambridge University Press.

Lee, I. A., \& Preacher, K. J. (2013). Calculation for the test of the difference between two dependent correlations with one variable in common [Computer software]. Available from http://quantpsy.org.

Li, N. P., Lim, A. J., Tsai, M. H., \& Jiaqing, O. (2015). Too materialistic to get married and have children? PLoS ONE, 10(5), e0126543. https://doi.org/10.1371/journal.pone.0126543.

Martos, T., Szabó, G., \& Rózsa, S. (2006). Psychometric characteristics of the shortened aspiration index in Hungarian sample. Mentálhigiéné és Pszichoszomatika, 7(3), 171-191. https://doi.org/10.1556/ Mentál.7.2006.3.2.

Moller, A. C., Meier, B. P., \& Wall, R. D. (2010). Developing an experimental induction of flow: Effortless action in the lab. In B. Bruya (Ed.), Effortless attention: A new perspective in the cognitive science of attention and action (pp. 191-204). Cambridge, MA: MIT Press. https://doi.org/10.7551/mitpr ess/9780262013840.003.0010.

Opree, S. J., Buijzen, M., van Reijmersdal, E. A., \& Valkenburg, P. M. (2014). Children's advertising exposure, advertised product desire, and materialism: A longitudinal study. Communication Research, 14(5), 717-735. https://doi.org/10.1177/0093650213479129.

Patall, E. A., Cooper, H., \& Robinson, J. C. (2008). The effects of choice on intrinsic motivation and related outcomes: A meta-analysis of research findings. Psychological Bulletin, 134(2), 270-300. https://doi. org/10.1037/0033-2909.134.2.270.

Pekrun, R., Goetz, T., Daniels, L. M., Stupnisky, R. H., \& Perry, R. P. (2010). Boredom in achievement settings: Exploring control-value antecedents and performance outcomes of a neglected emotion. Journal of Educational Psychology, 102(3), 531-549. https://doi.org/10.1037/a0019243.

Richins, M. L. (2004). The material values scale: Measurement properties and development of a short form. Journal of Consumer Research, 31(1), 209-219. https://doi.org/10.1086/383436.

Richins, M. L., \& Dawson, S. (1992). A consumer values orientation for materialism and its measurement: Scale development and validation. Journal of Consumer Research, 19(3), 303-316. https://doi. org/10.1086/209304.

Roberts, J. A., \& Clement, A. (2007). Materialism and satisfaction with over-all quality of life and eight life domains. Social Indicators Research, 82(1), 79-92. https://doi.org/10.1007/s11205-006-9015-0.

Rogatko, T. P. (2009). The influence of flow on positive affect in college students. Journal of Happiness Studies, 10(2), 133-148. https://doi.org/10.1007/s10902-007-9069-y.

Rokeach, M. (1973). The nature of human values. New York: Free Press.

Ryan, R. M., \& Deci, E. L. (2000). Self-determination theory and the facilitation of intrinsic motivation, social development, and well-being. American Psychologist, 55(1), 68-78.

Sahoo, F. M., \& Sahu, R. (2009). The role of flow experience in human happiness. Journal of the Indian Academy of Applied Psychology, 35, 40-47.

Schroeder, J. E., \& Dugal, S. S. (1995). Psychological correlates of the materialism construct. Journal of Social Behavior and Personality, 10(1), 243-253.

Schwartz, S. H. (1992). Universals in the content and structure of values: Theory and empirical tests in 20 countries. In M. Zanna (Ed.), Advances in experimental social psychology (Vol. 25, pp. 1-65). New York: Academic Press. https://doi.org/10.1016/S0065-2601(08)60281-6.

Schwartz, S. J., \& Waterman, A. S. (2006). Changing interests: A longitudinal study of intrinsic motivation for personally salient activities. Journal of Research in Personality, 40(6), 1119-1136. https://doi. org/10.1016/j.jrp.2005.12.003.

Seeman, M. (1959). On the meaning of alienation. American Sociological Review. https://doi. org/10.2307/2088565. 
Seligman, M. E. (2012). Flourish: A visionary new understanding of happiness and well-being. London: Nicholas Brealey.

Sheldon, K. M., \& Kasser, T. (1995). Coherence and congruence: Two aspects of personality integration. Journal of Personality and Social Psychology, 68(3), 531-543. https://doi. org/10.1037/0022-3514.68.3.531.

Sheldon, K. M., \& Kasser, T. (2008). Psychological threat and extrinsic goal striving. Motivation and Emotion, 32(1), 37-45. https://doi.org/10.1007/s11031-008-9081-5.

Sheldon, K. M., \& Krieger, L. S. (2014). Walking the talk: Value importance, value enactment, and wellbeing. Motivation and Emotion, 38(5), 609-619. https://doi.org/10.1007/s11031-014-9424-3.

Somer, E., \& Ruvio, A. (2014). The going gets tough, so let's go shopping: On materialism, coping, and consumer behaviors under traumatic stress. Journal of Loss and Trauma, 19(5), 426-441. https://doi. org/10.1080/15325024.2013.794670.

Stenseng, F., Rise, J., \& Kraft, P. (2012). Activity engagement as escape from self: The role of self-suppression and self-expansion. Leisure Sciences, 34(1), 19-38. https://doi.org/10.1080/01490400.2012.63384 9.

Swinyard, W. R., Kau, A. K., \& Phua, H. Y. (2001). Happiness, materialism, and religious experience in the US and Singapore. Journal of Happiness Studies, 2(1), 13-32. https://doi.org/10.1023/A:1011596515 474.

Ullén, F., de Manzano, Ö., Almeida, R., Magnusson, P. K., Pedersen, N. L., Nakamura, J., et al. (2012). Proneness for psychological flow in everyday life: Associations with personality and intelligence. Personality and Individual Differences, 52(2), 167-172. https://doi.org/10.1016/j.paid.2011.10.00.

United Nations. (2015). Transforming our world: The 2030 agenda for sustainable development. New York: UN Publishing.

Weinstein, N., Przybylski, A. K., \& Ryan, R. M. (2009). Can nature make us more caring? Effects of immersion in nature on intrinsic aspirations and generosity. Personality and Social Psychology Bulletin, 35(10), 1315-1329. https://doi.org/10.1177/0146167209341649.

Whelan, J., \& Hingston, S. (2016). The bright side to cuing consumerism: Consumer cues make individuals with low childhood socioeconomic status more prosocial. In P. Moreau \& S. Puntoni (Eds.), NA-Advances in consumer research (Vol. 44, pp. 677-678). Duluth, MN: Association for Consumer Research.

Publisher's Note Springer Nature remains neutral with regard to jurisdictional claims in published maps and institutional affiliations. 